\title{
A rehabilitation program for lung cancer patients during postthoracotomy chemotherapy
}

This article was published in the following Dove Press journal:

OncoTargets and Therapy

10 March 2014

Number of times this article has been viewed

\section{Amy J Hoffman' \\ Ruth Ann Brintnall ${ }^{2}$ \\ Alexander von $\mathrm{Eye}^{3}$ \\ Lee W Jones ${ }^{4}$ \\ Gordon Alderink ${ }^{5}$ \\ Lawrence H Patzelt ${ }^{6}$ \\ Jean K Brown ${ }^{7}$}

'College of Nursing, Michigan State University, East Lansing, MI, USA;

${ }^{2}$ Kirkhof College of Nursing, Grand Valley State University, Grand Rapids, MI, USA; '3Psychology Department, Michigan State University, East Lansing, MI, USA; ${ }^{4}$ Duke Center for Cancer Survivorship Department of Radiation Oncology, Duke University Medical Center, Durham, NC, USA;

${ }^{5}$ Frederik Meijer Honors College, Grand Valley State University, Grand Rapids, MI, USA; ${ }^{6}$ Spectrum Health, Grand Rapids, MI, USA and College of Human Medicine, Michigan State University, East Lansing, MI, USA; ${ }^{7}$ School of Nursing, University at Buffalo, the State University of New York, Buffalo, NY, USA
Correspondence: Amy J Hoffman College of Nursing, Michigan State University, Office C246 Bott Building for Nursing Education and Research, I 355 Bogue Street, East Lansing, MI, USA 488241317

$\mathrm{Tel}+\mathrm{I} 6168267820$

Fax +I 6166825373

Email amy.hoffman@ht.msu.edu
Objective: The objective of this pilot study was to describe the effects of a 16-week home-based rehabilitative exercise program on cancer-related fatigue (CRF), other symptoms, functional status, and quality of life (QOL) for patients with non-small cell lung cancer (NSCLC) after thoracotomy starting within days after hospital discharge and continuing through the initiation and completion of chemotherapy.

Materials and methods: Five patients with NSCLC completed the Brief Fatigue Inventory (measuring CRF severity) and the MD Anderson Symptom Inventory (measuring symptom severity) before and after thoractomy, and at the end of each week of the 16-week exercise program. Additionally, the Medical Outcomes Study Short Form-36 (measuring physical and mental functional status) and the Quality of Life Index (measuring QOL) were completed before and after thoracotomy, after weeks 3, 6, 12, and 16 (the end of the exercise program). Further, the 6-minute walk test (measuring functional capacity) was administered before thoracotomy, prior to the initiation of chemotherapy and/or radiation therapy, and at the end of the 16-week exercise program, after completion of chemotherapy.

Results: Participants had a mean age of 63 years and a mean of five comorbid conditions; the exercise program was initiated within 4 days after hospital discharge. Participants' CRF severity scores were reduced to mild levels, while the mean number of symptoms decreased from 9 postthoracotomy to 6 after the exercise program, with mean levels of severity and interference decreasing to below prethoracotomy levels. Likewise, participants' functional status and QOL after completing the exercise program improved to near or above prethoracotomy levels.

Conclusion: The home-based, light-intensity exercise program for NSCLC patients receiving and completing adjuvant chemotherapy postthoracotomy showed promising trends in improving CRF severity, other symptom severity, functional status, and QOL. Further testing via a two-arm randomized controlled trial is being conducted.

Keywords: lung cancer, exercise, cancer-related fatigue, symptoms, functional status, quality of life

\section{Background}

Innovations in surgical techniques and combined therapies have extended the lives of patients with localized non-small cell lung cancer (NSCLC) to a 5-year survival rate of $52 \% .{ }^{1}$ Even so, survivorship presents with complex needs and high symptom burden originating from the negative consequences of cancer, its treatment, and other comorbidities. ${ }^{2}$ Unfortunately, symptom burden is often accompanied by poor symptom control, yielding greater unmet supportive care needs. ${ }^{3}$ In response, the American College of Chest Physicians has updated its lung cancer guidelines, emphasizing symptom management by noting that symptoms are as important to treat 
as the cancer itself. ${ }^{4}$ In addition, at an Institute of Medicine National Cancer Policy Forum workshop, Dr Betty Ferrell, a nurse researcher from the City of Hope National Medical Center, noted that "symptom management has not received sufficient attention in developing survivorship care plans." 5 Thus, to preserve and maximize functional status and quality of life (QOL), symptom management plans should be initiated in a patient-health care team setting. Likewise, these plans should be initiated at diagnosis, with reassessment and evaluation throughout the various transitions of the cancer survivorship trajectory to promote self-management of symptoms. ${ }^{6}$ The purpose of this article is to describe a pilot study that examined the effects of a rehabilitation exercise program targeting cancer-related fatigue (CRF), other symptoms, functional status, and QOL for postthoracotomy NSCLC patients receiving chemotherapy.

Knowing that the symptom management need was great for the postsurgical NSCLC population, our team set out to improve our understanding of fatigue management during key transitions of the postthoracotomy NSCLC survivorship trajectory. Cancer-related fatigue is a common, severe, and distressing problem in the NSCLC population and is highly prevalent, with one survey reporting that $80 \%$ of 1,569 cancer patients undergoing chemotherapy and/or radiation therapy reported $\mathrm{CRF} .^{7}$ Cancer-related fatigue is associated with further negative effects on other symptoms, physical functioning, and QOL. ${ }^{8,9}$ The specific mechanisms underlying CRF have not been determined, but it is known that the effect of exercise on CRF has been positive. ${ }^{10}$ Consequently, experts recommend beginning the assessment of CRF at cancer diagnosis and that assessment continue regularly throughout the cancer survivorship trajectory. ${ }^{10}$ Likewise, experts urge that rehabilitation targeting CRF should start at cancer diagnosis and continue even after completion of cancer treatment. ${ }^{10}$ However, little research exists on how best to provide rehabilitative exercise for the postthoracotomy NSCLC population throughout recovery and chemotherapy. ${ }^{11}$ As a result, our multidisciplinary research team was guided by a synthesis of the Theory of Symptom Self-Management ${ }^{12}$ and the Transitional Care Model $^{13}$ to develop and implement a home-based, lightintensity exercise program for postthoracotomy NSCLC patients. The exercise program was initiated immediately upon transition from hospital to home and continued for 16 weeks through the completion of chemotherapy. To our knowledge, this study is the first to address the rehabilitation and symptom self-management voids experienced by postthoracotomy NSCLC patients through their immediate postsurgical recovery period and subsequent chemotherapy and/or radiation therapy.

\section{Methods \\ Design, setting, and participants}

This pilot study was approved by the Human Research Protection Programs at Michigan State University, Grand Valley State University, and an affiliated university teaching hospital in Michigan. Patients were invited to participate if they were 21 years of age or older, had a suspected diagnosis of NSCLC, had stable comorbidities, and had a prethoracotomy Karnofsky Performance Status score of 70\%. Other eligibility criteria included confirmation of NSCLC postthoracotomy, medical clearance to participate in the exercise program, and a willingness to follow health and safety procedures. Patients were excluded if they had severe deficits in vision, hearing, or speaking; used oxygen for daily living; weighed more than 300 pounds; had a history of seizures or dementia; or had conditions preventing safe participation in the exercise program. This article describes the experience of the patients who underwent thoracotomy followed by adjuvant chemotherapy beginning about 5 weeks after the start of the exercise program.

\section{Study procedures}

An oncology registered nurse recruited and facilitated enrollment of patients, and research staff collected data. After informed consent was obtained, baseline prethoracotomy data were collected, including demographics and measurements of CRF severity, severity of other symptoms, functional status, and QOL. These data were collected again postthoracotomy, within 72 hours of discharge from the hospital. At the end of exercise program weeks $1-16, \mathrm{CRF}$ and other symptom data were collected via telephone by research staff. At weeks 3, 6,12 , and 16 , patients completed functional status and QOL measures at home and mailed them back to research staff. A 6-minute walk test (6MWT) was conducted on three occasions: 1) prethoracotomy (T1); 2) prior to receiving chemotherapy and/or radiation therapy (T2); and 3) after 16 weeks on the exercise program, at the completion of chemotherapy and/or radiation therapy (T3).

\section{Exercise program}

After enrollment and prior to thoracotomy, patients received fatigue self-management education to support the exercise program. This educational material was based on National Comprehensive Cancer Network Guidelines and was taught by a registered nurse. The exercise program was initiated within 4 days of hospital discharge, with an assessment completed by 
the nurse to ensure that core symptoms (pain, dyspnea, nausea, and vomiting) were managed. Once symptom management was ensured, the nurse completed the first home visit to implement the light-intensity (less than 3.0 metabolic equivalents) exercise program, which consisted of walking and balance exercises using the Nintendo Wii (Nintendo, Redmond, WA, USA). The program included walking initiated at 5 minutes a day for 5 days during week 1 of the program with the goal of walking 30 minutes a day for 5 days by week 6 . The patient's walking duration was increased 5 minutes per day each week if the patient's perceived self-efficacy for achieving that duration was $70 \%$ or greater on a $0 \%-100 \%$ scale, with $100 \%$ meaning high perceived self-efficacy. Once a patient reached 30 minutes a day, walking duration was increased by $10 \%$ per week if the patient desired to increase his or her walking duration.

In addition, patients incorporated balance exercise derived from the Nintendo Wii 5 days a week for 16 weeks. Patients started exercising at the nurse's first home visit after discharge from the hospital. After the first home visit the nurse made a follow-up phone call to answer any questions about the program. The nurse made a second home visit at the start of week 2 to adjust the exercise prescription; this visit was followed by phone visits at the start of weeks 3-16. Exercise prescriptions were modified when needed for situations such as surgery for the placement of a port for chemotherapy and/ or needing to travel to attend events (eg, weddings). The nurse provided weekly support by reviewing each patient's exercise progress, providing fatigue self-management interventions to support exercise, and adjusting the exercise prescription. Likewise, an exercise program safety plan continued throughout the 16 weeks of the study. ${ }^{14}$

\section{Main outcome measures}

Patient characteristics were obtained through the use of a demographic questionnaire, a modified comorbidity questionnaire, a medical chart review, and a self-report of exercise behavior. The instruments used in this study, described below, have established reliability and validity.

CRF severity was measured using the Brief Fatigue Inventory. ${ }^{15}$ Patients rated nine items on a $0-10$ scale $(10=$ most severe), depicting CRF severity and interference at its worst in the past 24 hours.

Symptom severity and interference were measured with the MD Anderson Symptom Inventory score and lung module (MDASI). ${ }^{16}$ The MDASI assesses the severity of 16 symptoms and six items measuring the interference from these symptoms on daily life, all at their worst in the past 24 hours. Because the Brief Fatigue Inventory was used to measure fatigue, the
MDASI fatigue item was excluded from this assessment. Thus, 15 symptoms and six interference items were rated on a $0-10$ scale $(10=$ most severe; most interference).

Functional status was measured via two dimensions: functional capacity and functional performance. Functional capacity was measured using the 6MWT, which was implemented as per American Thoracic Society Guidelines. ${ }^{17}$ The 6MWT is an assessment of submaximal functional capacity measured in distance walked in meters in 6 minutes and in associated fatigue levels. Fatigue levels were measured using the modified Borg Scale, a scale that has been used successfully in the postthoracotomy NSCLC population, measuring fatigue severity on a $0-10$ scale $(10=$ most severe $) .{ }^{17}$ The $6 \mathrm{MWT}$ was chosen to measure functional capacity because of its relevance in measuring functional capacity to perform everyday activities. ${ }^{18,19}$ Also, according to the American Thoracic Society, ${ }^{17}$ the $6 \mathrm{MWT}$ is an appropriate tool to assess the response to treatment for patients with moderate or severe lung disease, including NSCLC, and 6MWT performance is also a strong independent predictor of mortality in patients with lung disease, including NSCLC. ${ }^{20}$ Further, Jones et $\mathrm{al}^{20}$ used the 6MWT in their study and found that patients with NSCLC who walked $>450$ meters were considered high functioning and had significantly prolonged survival relative to patients walking $<450$ meters.

With the 6MWT providing insight into functional capacity, the Medical Outcomes Study Short Form-36 Version 2 Acute Recall (SF-36) was selected to measure functional performance. The SF-36 is a widely used instrument that measures eight subscales encompassing the physical and mental health components of functional status. The scores of the subscales are linearly transformed, producing normative scores ranging from $0-100$, with higher scores indicating better functional status.

QOL was measured using the Quality of Life Index (QLI). ${ }^{21-23}$ The QLI measures satisfaction with various aspects of life (32 items) and the importance of each aspect of life ( 32 items) to the person. The assessment produces an overall QOL score consisting of four domains: health and functioning; psychological and spiritual; socioeconomic; and family. The QLI is rated on a 6-point scale ranging from very dissatisfied to very satisfied for the satisfaction domain, and from very unimportant to very important for the importance domain. Scores for the QLI range from 0 (worst) to 30 (best), with higher scores specifying greater QOL.

\section{Data analysis}

Data were analyzed using SPSS statistical software, version 19.0 (IBM Corporation, Armonk, NY, USA). 
Descriptive statistics were used to calculate means and standard deviations to depict study results. The pattern of change in CRF and symptom outcomes were graphed at baseline before and after thoracotomy, and at the end of each week for 16 weeks. Results for functional status (performance) and QOL outcomes were graphed at baseline, prethoracotomy, and postthoracotomy, and at the end of weeks 3, 6, 12, and 16 of the study. Likewise, trends in mean scores for functional status (capacity) were depicted to show change across three time points (T1, T2, and T3).

\section{Results}

Thirteen patients were invited to participate in this pilot study and seven agreed to be enrolled. All seven patients completed the 16-week exercise program. Five of the seven patients (three women and two men) received adjuvant chemotherapy and were included in our analysis.

\section{Patient characteristics}

Certain patient characteristics are shown in Table 1. The five patients were Caucasian, married, had a mean age of 63.4 \pm 7.3 years (range, 53-73 years), and underwent lobectomy for stage IIA-IIIA NSCLC. Final histology showed four patients with adenocarcinoma and one with squamous carcinoma. All five had a high school education, with three having graduated from college. Four patients were employed outside the home, and one was a full-time homemaker. All patients reported a history of smoking, with all quitting at the time of diagnosis of NSCLC and throughout the duration of the study.

A mean presurgical Karnofsky Performance Status score of $70 \%$ was assessed in two patients, meaning that they were unable to perform normal work activity; three patients were assessed with a score of $90 \%$, meaning that they showed signs and symptoms of minor disease and reported minimal interference with normal activity. Patients had a mean of $5.4 \pm 4.1$ controlled comorbidities (range, 2-12 comorbidities), the most common of which were chronic obstructive pulmonary disease, previous cancer, cardiac disease, hypertension, and arthritis, including osteoarthritis and rheumatoid arthritis. Mean length of stay in the hospital was $8.4 \pm 2.6$ days (range, 5-12 days). Three patients experienced postoperative complications prior to discharge, including atrial arrhythmias, hemorrhage requiring surgical correction, and atelectasis.

On average, chemotherapy started 42 days postthoracotomy, approximately 5 weeks after initiation of the exercise program. All patients underwent chemotherapy with a platinum compound, either cisplatin or carboplatin combined

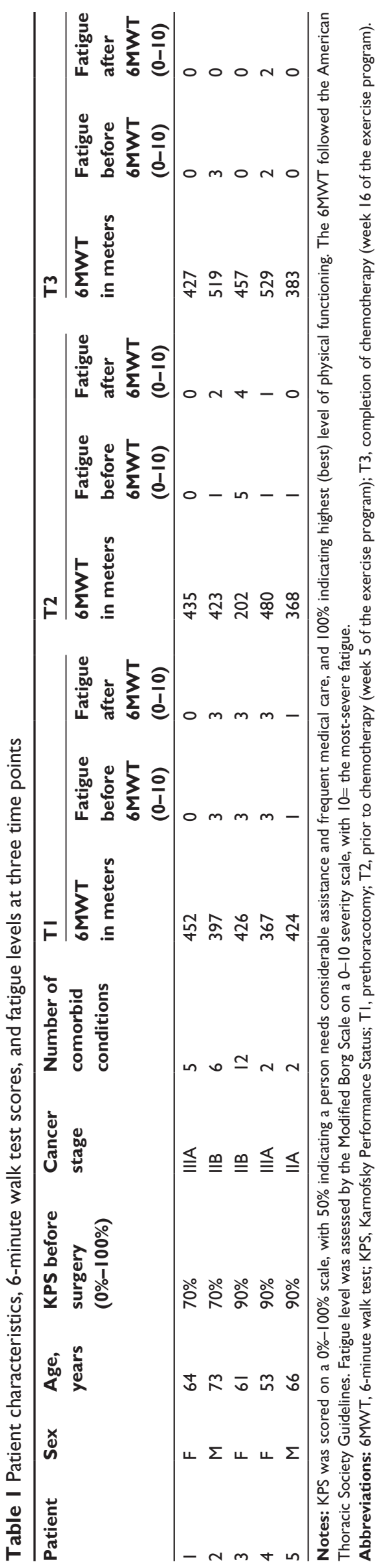


with another agent (paclitaxel; pemetrexed, vinorelbine, or gemcitabine HCL). One patient also received radiation therapy alongside chemotherapy 5 days a week (for a total of 28 treatments) for stage IIIA NSCLC.

On average, patients started exercising $64 \pm 28$ hours after discharge from the hospital, at the nurse's first home visit. The exercise program safety plan continued throughout the 16 weeks of the study with no adverse events. Patients achieved a mean of $24.0 \pm 4.3$ minutes walking for 5 days during week 6. At week 15, a mean walking score of $31.0 \pm 6.5$ minutes was achieved by all five patients. At week 16 , four patients achieved a mean of $36.0 \pm 5.0$ minutes, with the fifth patient not participating due to a flare-up of rheumatoid arthritis in her lower extremities at the start of week 16. The overall adherence to the walking intervention for weeks $1-16$ was $92.6 \% \pm 4.6 \%$ (range, $87.0 \%-98.1 \%$ ). The overall adherence to the balance intervention for weeks 1-16 was $94.7 \% \pm 7.0 \%$ (range, $82.5 \%-100 \%$ ).

\section{CRF severity and other symptoms}

Figure 1 displays the overall pattern of mean symptom scores from prethoracotomy to postthoracotomy through week 16 of the exercise program. On average, participants experienced seven symptoms prethoracotomy, nine symptoms postthoracotomy, seven symptoms at week 4 of the exercise program, and six symptoms at week 16 of the program. CRF severity before and after thoracotomy remained stable at 3.2 and 3.3 on a $0-10$ scale, respectively, and was 4.2 in week 2 of the exercise program and 1.5 at week 16 . The severity of other symptoms (composite score) increased from 3.6 prethoracotomy to 4.7 postthoracotomy, decreased to 2.7 at week 5 prior to the start of chemotherapy, increased to 3.8 at week 12 during chemotherapy, and decreased to 2.0 at week 16. Interference from other symptoms increased from 2.1 prethoracotomy to 3.3 postthoracotomy, increased to 3.9 at the end of week 1 , and subsequently decreased throughout chemotherapy to 1.5 at week 16 .

\section{Functional status (functional capacity)}

The 6MWT distance is used to assess level of functioning where $>450$ meters defines high functioning. One out of five participants qualified as high functioning prethoracotomy and prior to chemotherapy. Three out of five participants qualified as high functioning postintervention ( $40 \%$ improvement over prethoracotomy and prior to chemotherapy levels).

The mean score for the 6MWT distance prethoracotomy (T1) was $413 \pm 32$ meters, and scores decreased by 31 meters or $8 \%$ to a distance of $382 \pm 108$ meters prior to the start of chemotherapy (T2). However, the 6MWT distance improved by 81 meters, a $21 \%$ increase, to $463 \pm 62$ meters from T2 to the conclusion of the exercise program and chemotherapy at week 16 (T3). Moreover, this post-exercise-program increase in the 6MWT distance scores also exceeded prethoracotomy scores by 50 meters, or $12 \%$.

The post-6MWT mean Borg fatigue severity score decreased from $2.0 \pm 1.4$ at $\mathrm{T} 1$ to $1.4 \pm 1.7$ at $\mathrm{T} 2$. Likewise, the post-6MWT Borg fatigue severity score continued to decrease to a mean severity level of $0.4 \pm 0.9$ at $\mathrm{T} 3$.

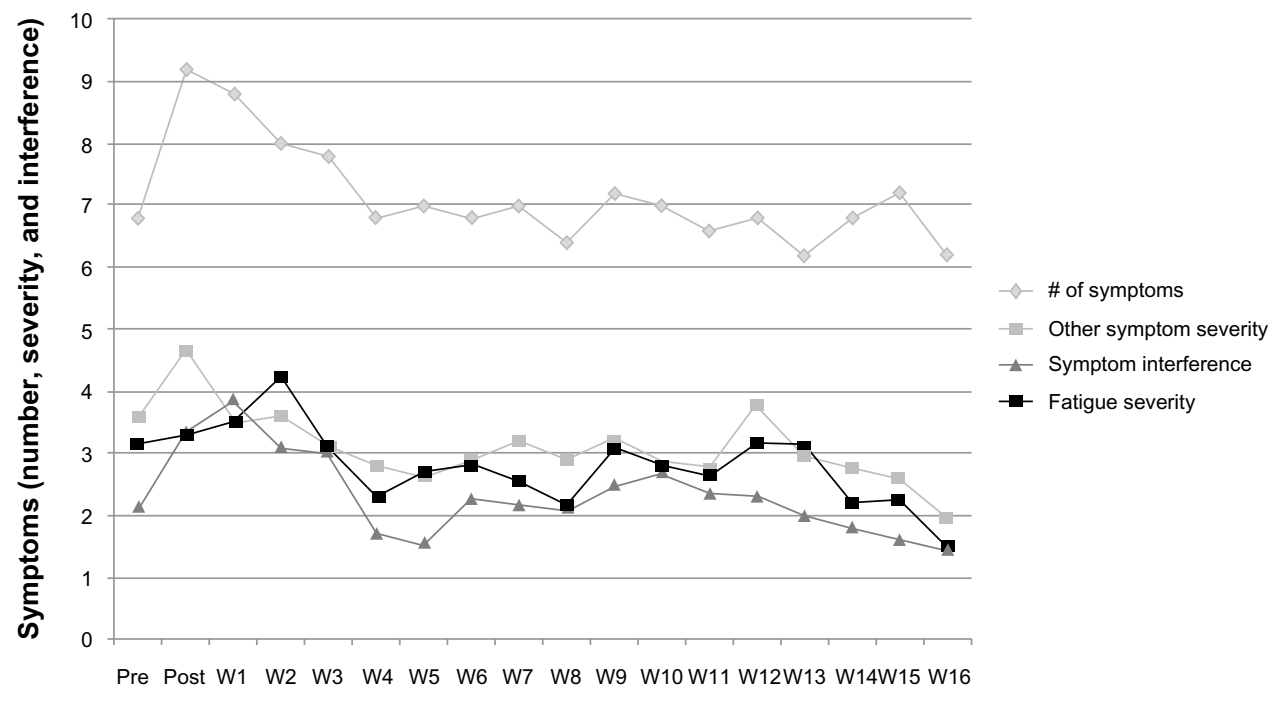

Figure I Mean number, severity, and interference from symptoms before and after thoracotomy and for weeks $\mathrm{I}-16$ after the start of the exercise intervention. Notes: Symptom severity and interference were measured on the 0-10 scale of the MDASI, with higher scores indicating greater severity and interference. Cancer-related fatigue severity was measured on the $0-10$ scale of the BFI, with higher scores indicating greater severity.

Abbreviations: BFI, Brief Fatigue Inventory; MDASI, MD Anderson Symptom Inventory; Post, postthoracotomy; Pre, prethoracotcomy; W, week. 
Table 1 provides a summary of each patient's rehabilitation progress with pertinent clinical information and corresponding 6MWT scores. Patients 1 and 5 nearly maintained their T1 functional capacity at T3 (94\% and 90\%, respectively), while showing stability and improvement in their Borg fatigue severity scores. Moreover, patients 2, 3, and 4 improved their functional capacity, as their 6MWT distance scores at T3 increased over T1 scores by 31\%, 7\%, and $44 \%$, respectively, while all improved their Borg fatigue severity scores as well.

\section{Functional status (functional performance) Physical functional status}

As depicted in Figure 2, the SF-36 physical health component score, indicative of overall physical functional status prethoracotomy, was 50.9 , worsened to 31.5 postthoracotomy, and improved to 43.5 and 47.3 at weeks 3 and 6 , respectively. During midchemotherapy treatment at week 12 , the physical health component score declined to 43.6 and recovered to 46.8 at the end of chemotherapy at week 16 .

\section{Mental functional status}

As depicted in Figure 2, the SF-36 mental health component mean score, indicative of mental functional status, was 43.8 prethoracotomy. Postthoracotomy and at weeks 3, 6, 12 and 16, the mental health component mean scores showed an increasing trend to $46.1,47.9,51.1,51.0$, and 53.2, respectively.

\section{Quality of life}

As depicted in Figure 3, overall QOL scores worsened from 24.0 prethoracotomy to 22.3 postthoracotomy. Overall QOL increased to 25.2 in week 3, decreased to 23.3 and 22.3 in weeks 6 and 12 during chemotherapy, respectively, and increased to 25.9 at the end of week 16, exceeding pre-thoracotomy levels. Health and functioning domain scores decreased from 20.8 prethoracotomy to 16.8 postthoracotomy. Week 3 saw scores increase to 22.0, only to decline during chemotherapy to 20.2 at week 6 and 18.9 at week 12 , returning to above-prethoracotomy levels, to 23.7 , at week 16. Socioeconomic domain scores increased from a prethoracotomy level of 26.1 to postthoracotomy and week-3 levels of 26.3 and 27.2, respectively. A decrease in scores occurred during chemotherapy at weeks 6 and 12 to 25.7 and 25.4, respectively, returning at week 16 to 27.3, above prethoracotomy levels. Psychological and spiritual domain scores followed the same pattern of increase and decrease as the socioeconomic domain, with scores at prethoracotomy and postthoracotomy and weeks 3, 6, 12 and 16 of 26.2, 24.6, 27.9, 24.3, 24.2, and 27.2, respectively, again with post-exercise-program levels trending above prethoracotomy levels. Family domain scores increased from 26.0 prethoracotomy to 26.8 postthoracotomy and remained stable after weeks 3 and 6 at 26.7 and 26.2, respectively. At week 12, the family domain score decreased to 23.7 and returned to 27.6 at week 16 , above prethoracotomy levels.

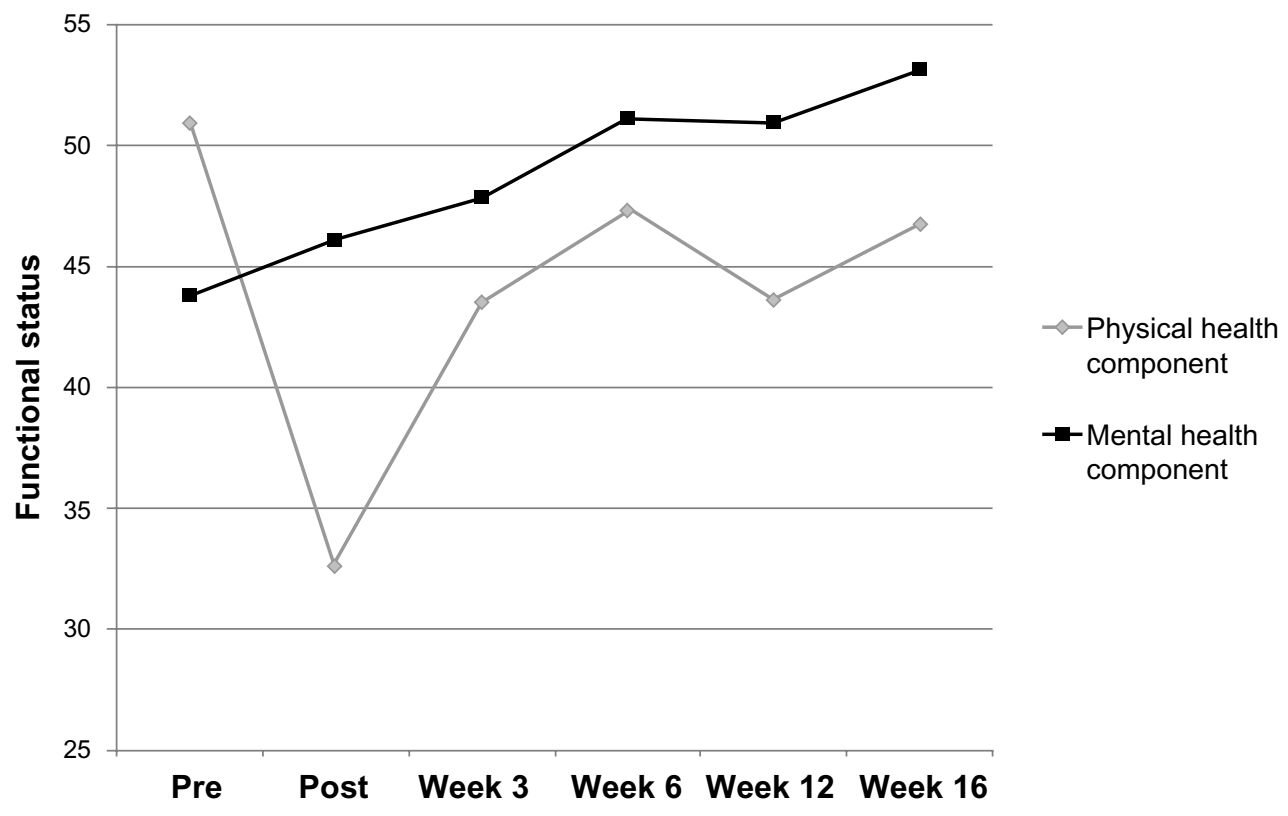

Figure 2 Functional status before and after thoracotomy, and at weeks 3, 6, 12, and 16 after the start of the exercise intervention.

Notes: Functional status was measured using both the physical and mental health components of the SF-36; possible scores ranged from 0-100, with higher scores indicating greater (better) levels of functional status.

Abbreviations: Post, postthoracotomy; Pre, prethoracotomy; SF-36, Medical Outcomes Study Short Form-36 Version 2 Acute Recall. 


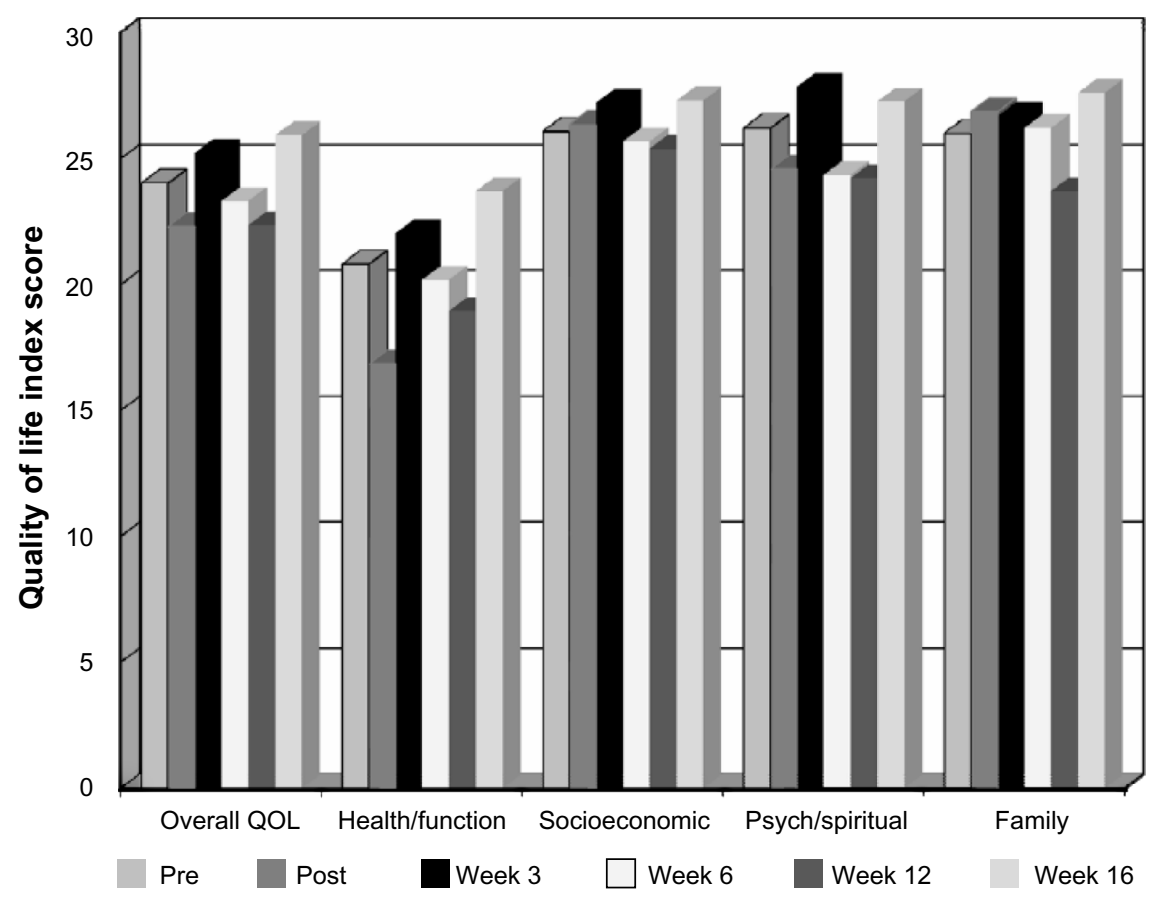

Figure 3 Quality of life before and after thoracotomy, and at weeks 3, 6, 12, and 16 after the start of the exercise intervention. Note: QOL was measured on the 0-30 scale of the QLI, with higher scores indicating better quality of life.

Abbreviations: QLI, Quality of Life Index; QOL, quality of life; Post, posthoracotomy; Pre, prethoracotomy; Psych, psychological.

\section{Discussion}

To our knowledge, this is the first study to examine the effects of a home-based, self-managed exercise program initiated within days of hospital discharge postthoracotomy for NSCLC and continued through recovery that includes adjuvant chemotherapy and radiation therapy. Despite the fact that patients were older, had a high number of comorbidities, had decreased performance status prior to surgery, and experienced thoracotomy-related complications, they showed improvement in the severity of CRF and other symptoms, functional status, and QOL over and above prethoracotomy baseline measurements. It should be noted that other studies have reported multiple symptoms postthoracotomy in this patient population characterized with multiple comorbidities. ${ }^{2,9,24-26}$ However, this paper adds to the literature because it demonstrates consistent stabilization of CRF and other symptoms in NSCLC patients to mild severity levels. Further, this was accomplished during the initial surgical recovery phase and was extended through completion of chemotherapy with a home-based, light-intensity exercise program starting the first week after discharge from the hospital.

Likewise, to our knowledge this is the first study to report improvements in functional status very early in the recovery trajectory of NSCLC patients after starting the exercise program. Patients recovered to $92 \%$ of their prethoracotomy functional capacity, with less report of fatigue, after
5 weeks of exercise and prior to starting chemotherapy. The study realized a $40 \%$ improvement (postintervention versus presurgery) in the number of participants categorized in the high functioning group ( $>450$ meters covered in the 6MWT). Since studies have shown that high functioning is a significant predictor of survival, these results are promising. ${ }^{20}$ Achieving increased functional capacity with a corresponding decrease in fatigue is also promising and supports positive exerciseprogram outcomes on endurance and stamina.

Relative to functional performance, while the SF-36 physical health component dropped significantly, as expected, after surgery, it recovered to near presurgical levels even after patients completed chemotherapy and/or radiation. This may be attributed to initiating physical activity immediately upon return home from the hospital and continuing throughout recovery. Similarly, the SF-36 mental health component showed a steady increase, finishing at a level above prethoracotomy and the US general population. ${ }^{27}$ This increase in mental health component scores throughout the exercise program period is promising and may correspond to the fact that these patients were equipped with an effective means of managing their symptoms throughout the recovery trajectory. Although there are few reports of the functional status in the NSCLC population before and immediately after thoracotomy, the findings in this study affirm those reports..$^{25,28}$ However, this paper adds to the literature by longitudinally 
describing two dimensions of functional status: functional capacity via the $6 \mathrm{MWT}$ and functional performance via the SF-36. For both functional capacity and functional performance, the implementation of the exercise program immediately after hospital discharge has shown promising improvement over the NSCLC treatment trajectory, including thoracotomy and chemotherapy. ${ }^{29}$

Finally, as expected, overall QOL and its respective domains followed a pattern similar to functional status. The results of the study were promising in that QOL levels were sustained at an adequate level throughout chemotherapy, while scores improved over prethoracotomy levels upon completion of the program. This may be a result of increased mental and physical health due to incorporation of the light exercise program. Because very little is known about QOL over time in patients who have undergone thoracotomy and subsequent treatment with chemotherapy and/or radiation therapy, this study adds to the literature as it incorporates a distinct QOL measure in conjunction with symptoms and functional status assessment throughout the recovery trajectory. In addition, to our knowledge, this is the first pilot study to document consistent preliminary efficacy in CRF severity, symptom severity, functional status, and QOL when NSCLC patients participate in a light-intensity exercise program designed to target $\mathrm{CRF}$.

\section{Conclusion}

As we continue to extend the lives of those with NSCLC, improving symptom management, functional status, and QOL will continue to be a priority for these patients, especially those with localized disease. Living with the effects of NSCLC and its various treatments, including thoracotomy and chemotherapy, begins at diagnosis and continues for the duration of a survivor's life. ${ }^{30}$ NSCLC and its treatment is a major threat to a survivor's QOL, and the lack of symptom self-management rehabilitation strategies is a major concern for survivors and health care providers. Recognizing this current gap, the National Action Plan for Cancer Survivorship has set a goal to identify and prioritize supportive care needs and design strategies to improve QOL. ${ }^{31}$ As such, designing fatigue self-management strategies for persons with NSCLC through light-intensity exercise as described has shown promise in controlling fatigue and other symptoms with a positive impact on functional status and QOL. Teaching patients minimalbarrier, evidenced-based exercise during a serious illness such as NSCLC could play a critical part in empowering them to self-manage their symptoms as cancer survivors. ${ }^{32}$ Even though this study is currently being expanded by increasing the number of participants and comparing the exercise group with a usual-care group, the implications of this pilot are exciting and promising. Self-management through home-based, light-intensity exercise can have a wide-reaching impact on the rehabilitation and recovery of postthoracotomy lung cancer patients receiving chemotherapy.

\section{Acknowledgment}

Funding for this research was received from Michigan State University, College of Nursing (East Lansing, MI, USA).

\section{Disclosure}

The authors report no conflicts of interest in this work.

\section{References}

1. American Cancer Society. Cancer Facts and Figures 2013. American Cancer Society, Inc.: Atlanta, GA; 2013. Available from: http://www. cancer.org/acs/groups/content/@epidemiologysurveilance/documents/ document/acspc-036845.pdf. Accessed October 31, 2013.

2. Cleeland CS, Zhao F, Chang VT, et al. The symptom burden of cancer: Evidence for a core set of cancer-related and treatment-related symptoms from the Eastern Cooperative Oncology Group Symptom Outcomes and Practice Patterns study. Cancer. 2013;119(24):4333-4340.

3. Yun YH, Shon EJ, Yang AJ, et al. Needs regarding care and factors associated with unmet needs in disease-free survivors of surgically treated lung cancer. Ann Oncol. 2013;24(6):1552-1559.

4. Simoff MJ, Lally B, Slade MG, et al. Symptom Management in Patients with Lung Cancer: Diagnosis and Management of Lung Cancer. 3rd ed: American College of Chest Physicians evidence-based clinical practice guidelines. Chest. 2013;143(Suppl 5):e455S-e497S.

5. National Coalition for Cancer Survivorship, Institute of Medicine National Cancer Policy Forum. Implementing Cancer Survivorship Care Planning: Workshop Summary. Hewitt M, Ganz PA, editors. Washington, DC: The National Academies Press; 2006.

6. Hoffman AJ, Given BA, von Eye A, Gift AG, Given CW. Relationships among pain, fatigue, insomnia, and gender in persons with lung cancer. Oncol Nurs Forum. 2007;34(4):785-792.

7. Henry DH, Viswanathan HN, Elkin EP, Traina S, Wade S, Cella D. Symptoms and treatment burden associated with cancer treatment: results from a cross-sectional national survey in the US. Support Care Cancer. 2008;16(7):791-801.

8. Brown JK, Cooley ME, Chernecky C, Sarna L. A symptom cluster and sentinel symptom experienced by women with lung cancer. Oncol Nurs Forum. 2011;38(6):E425-E435.

9. Brunelli A, Socci L, Refai M, Salati M, Xiumé F, Sabbatini A. Quality of life before and after major lung resection for lung cancer: a prospective follow-up analysis. Ann Thorac Surg. 2007;84(2):410-416.

10. National Comprehensive Cancer Network. NCCN Clinical Practice Guidelines in Oncology (NCCN Guidelines ${ }^{\circledR}$ ): Cancer-Related Fatigue, Version 1.2013. Fort Washington, PA: National Comprehensive Cancer Network; 2012. Available from: http://www.nccn.org/professionals/ physician_gls/pdf/fatigue.pdf. Accessed November 1, 2013.

11. Granger C, Denehy L. Exercise interventions following surgery for non-small cell lung cancer (NSCLC): the need for future randomised controlled trials. Lung Cancer. 2010;70(2):228-229.

12. Hoffman AJ. Enhancing self-efficacy for optimized patient outcomes through the theory of symptom self-management. Cancer Nurs. 2013;36(1):E16-E26.

13. Naylor MD, Brooten D, Campbell R, et al. Comprehensive discharge planning and home follow-up of hospitalized elders: a randomized clinical trial. JAMA. 1999;281(7):613-620. 
14. Hoffman AJ, Brintnall RA, Brown JK, et al. Virtual reality bringing a new reality to postthoracotomy lung cancer patients via a home-based exercise intervention targeting fatigue while undergoing adjuvant treatment. Cancer Nurs. 2014;37(1):23-33.

15. Mendoza TR, Wang XS, Cleeland CS, et al. The rapid assessment of fatigue severity in cancer patients: use of the Brief Fatigue Inventory. Cancer. 1999;85(5):1186-1196.

16. Cleeland CS, Mendoza TR, Wang XS, et al. Assessing symptom distress in cancer patients: the MD Anderson Symptom Inventory. Cancer. 2000;89(7):1634-1646.

17. ATS Committee on Proficiency Standards for Clinical Pulmonary Function Laboratories. ATS statement: guidelines for the six-minute walk test. Am J Respir Crit Care Med. 2002;166(1):111-117.

18. Steele B. Timed walking tests of exercise capacity in chronic cardiopulmonary illness. J Cardiopulm Rehabil. 1996;16(1):25-33.

19. Jones LW, Peddle CJ, Eves ND, et al. Effects of presurgical exercise training on cardiorespiratory fitness among patients undergoing thoracic surgery for malignant lung lesions. Cancer. 2007;110(3):590-598.

20. Jones LW, Hornsby WE, Goetzinger A, et al. Prognostic significance of functional capacity and exercise behavior in patients with metastatic non-small cell lung cancer. Lung Cancer. 2012;76(2):248-252.

21. Ferrans CE, Powers MJ. Quality of life index: development and psychometric properties. ANS Adv Nurs Sci. 1985;8(1):15-24.

22. Ferrans CE, Powers MJ. Psychometric assessment of the Quality of Life Index. Res Nurs Health. 1992;15(1):29-38.

23. Ferrans CE. Development of a quality of life index for patients with cancer. Oncol Nurs Forum. 1990;17(Suppl 3):15-19; discussion 20-21.

24. Handy JR. Functional outcomes after lung cancer resection: who cares as long as you are cured? Chest. 2009;135(2):258-259.
25. Handy JR, Asaph JW, Skokan L, et al. What happens to patients undergoing lung cancer surgery? Outcomes and quality of life before and after surgery. Chest. 2002;122(1):21-30.

26. Sarna L, Cooley ME, Brown JK, Chernecky C, Elashoff D, Kotlerman J. Symptom severity 1 to 4 months after thoracotomy for lung cancer. Am $J$ Crit Care. 2008;17(5):455-467; quiz 468.

27. Bassett DR Jr, Wyatt HR, Thompson H, Peters JC, Hill JO. Pedometermeasured physical activity and health behaviors in U.S. adults. Med Sci Sports Exerc. 2010;42(10):1819-1825.

28. Möller A, Sartipy U. Associations between changes in quality of life and survival after lung cancer surgery. J Thorac Oncol. 2012;7(1): 183-187.

29. Cavalheri V, Tahirah F, Nonoyama M, Jenkins S, Hill K. Exercise training undertaken by people within 12 months of lung resection for non-small cell lung cancer. Cochrane Database Syst Rev. 2013;7: CD009955.

30. Reuben SH. President's Cancer Panel 2003-2004 Annual Report. Living Beyond Cancer: Finding a New Balance. Leffall LD, editor. Bethesda, MD: US Department of Health and Human Services, National Institutes of Health, National Cancer Institute; 2004.

31. Centers for Disease Control and Prevention, Lance Armstrong Foundation. A National Action Plan for Cancer Survivorship: Advancing Public Health Strategies. Atlanta, GA: Centers for Disease Control and Prevention; 2004. Available from: http://www.cdc.gov/cancer/survivorship/pdf/plan.pdf. Accessed January 4, 2014.

32. Poghosyan H, Sheldon LK, Leveille SG, Cooley ME. Health-related quality of life after surgical treatment in patients with non-small cell lung cancer: a systematic review. Lung Cancer. 2013;81(1):11-26.
OncoTargets and Therapy

\section{Publish your work in this journal}

OncoTargets and Therapy is an international, peer-reviewed, open access journal focusing on the pathological basis of all cancers, potential targets for therapy and treatment protocols employed to improve the management of cancer patients. The journal also focuses on the impact of management programs and new therapeutic agents and protocols on

\section{Dovepress}

patient perspectives such as quality of life, adherence and satisfaction. The manuscript management system is completely online and includes a very quick and fair peer-review system, which is all easy to use. Visit http://www.dovepress.com/testimonials.php to read real quotes from published authors. 